Review Article

\title{
HIV-2: an overview
}

\section{Mangala S. Borkar*, Akshay A. Kashid}

Department of Medicine, Government Medical College, Aurangabad, Maharashtra, India

Received: 15 October 2015

Accepted: 21 November 2015

\author{
*Correspondence: \\ Dr. Mangala S. Borkar, \\ E-mail: kargalman@gmail.com
}

Copyright: (C) the author(s), publisher and licensee Medip Academy. This is an open-access article distributed under the terms of the Creative Commons Attribution Non-Commercial License, which permits unrestricted non-commercial use, distribution, and reproduction in any medium, provided the original work is properly cited.

\begin{abstract}
HIV-2 is much less common compared to HIV-1, has to be confirmed by HIV-2 Western Blot test and is resistant to Efavirinz and Nevirapine. There are two HIV viruses, HIV-1 and HIV-2. HIV-2 is relatively rare in India. The clinical course of HIV-2 infection is slower, plasma HIV-2 RNA levels are lower as compared to HIV-1 infection, but once the illness progresses to AIDS, the course is similar to HIV-1. In few cases, there may be mixed infection with both HIV-1 and HIV-2, but the course of the illness is like in HIV-1 However ,even in mixed infections, one has to give the therapy as we would in isolated HIV-2 infection. In general it is accepted that therapy in cases of HIV-2 infection should be started before there is clinical progression. Studies recommend starting therapy when CD4 count drops below 500. HIV-2 is intrinsically resistant to non-nucleoside reverse transcriptase inhibitors. Therapy in HIV-2 patients should include two nucleoside reverse transcriptase inhibitors plus an HIV-2 active boosted protease inhibitor or integrase strand transfer inhibitors. Monitoring of CD4 cell counts and clinical improvement should be used to assess response to treatment. Drugs used in Government ART centres in India under the umbrella of NACO are Tenofovir 300mg + Lamivudine $300 \mathrm{mg}+$ Lopinavir $200 \mathrm{mg}+$ Ritonavir $50 \mathrm{mg}$ and are observed to be beneficial clinically in terms of weight gain, increase in CD4 levels, prevention and control of opportunistic infections and improved sense of well-being.
\end{abstract}

Keywords: HIV-2, Raltegravir, Ritonavir boosted Lopinavir

\section{INTRODUCTION}

Globally, HIV-1 is far commoner than HIV-2. HIV-2 is relatively rare in India. In 1986, the first case of HIV-2 was isolated from AIDS patients in West Africa, in 1987 in the United States and in 1991 in India, respectively. Initially, HIV-2 was confined to West Africa. It is postulated that HIV-1 came to humans from chimpanzees and gorillas, whereas sooty mangabees transmitted HIV2. 1

HIV-2 is said to be less infectious and parent to child transmission is rare. HIV-2 Western blot is the confirmatory test for HIV-2 infection. Rapid screening tests may give false positive results.
Both HIV-1 and HIV-2 have the same modes of transmission and are associated with similar opportunistic infections and AIDS. However,

1. In persons infected with HIV-2, immunodeficiency seems to develop more slowly and cases are milder.

2. Compared to HIV-1, those with HIV-2 are less infectious early in the course of infection.

3. Vertical transmission of the virus is much less common. Parent-to-child transmission is only about $4 \%$, including that through breast feeding.

4. Nevirapine or Efavirinz are ineffective in HIV-2 infection.

5. Reliable viral load measurement is not yet possible for HIV-2 infection, hence the patient's response has to be judged clinically and by CD4 counts. ${ }^{2}$ 
Universally accepted treatment guidelines are not available. $^{3}$ In India, HIV-2 kits were made available under the national AIDS control program in 2012 and confirmatory tests started being carried out as a routine in referral laboratories like NARI (National AIDS Research Institute) in 2013. HIV-2 is intrinsically resistant to nonnucleoside reverse transcriptase (NNRTI) drugs, Nevirapine and Efavirinz. The options available for firstline HIV-2 treatment are the second line drugs for HIV-1 i.e. Nucleoside reverse transcriptase inhibitors, NRTIszidovudine, lamivudine, tenofir or abacavir or boosted protease inhibitors (PI- based) regimes, using saquinavir, lopinavir, darunavir, or indinavir. Boosted PI regime has been suggested by the U.S. department of Health and Human services. ${ }^{1}$ Triple drug regimens using NRTIs have been recommended by WHO earlier, but have been found to be rather unsuccessful. ${ }^{3}$ Brower et al and Desbois et al have found that Lopinavir, Saquinavir and Darunavir are potent inhibitors of HIV-2 protease in isolates. Atazanovir is not that effective. ${ }^{4}$ Experts favour the use of 2 NRTIs like tenofovir with lamivudine or emtricitabine plus a ritonavir boosted PI. ${ }^{1}$ The ANRS CO5 HIV-2 Cohort assessed treatment response in 29 HIV-2 positive individuals in whom lopinavir/ritonavir with NRTI showed improvement in CD4 count over 2 years in 20 patients. In these, HIV-RNA became undetectable too. The 2008 French guidelines state that atazanavir, fosamprenavir, and tipranavir should be used with caution in patients with HIV-2 infection. Among the newer antiretroviral agents, HIV-2 appears to be susceptible to the 2 integrase strand transferase inhibitors, raltegravir and elvitegravir. In the Government set-up, we use the regimen prescribed by NACO i.e. second-line ART.

Patients presenting to Integrated Counselling and Treatment Centre are subjected to 3 rapid tests, of which the second picks up HIV-2 positivity. This has to be confirmed by Western Blot for HIV-2 and PCR that is performed in Maharashtra at NARI (National Aids Research Institute), Pune. When a case tests positive for HIV-2 in ICTC, he is referred to ART center, from where this case is referred to NARI. Fresh blood sample is drawn at NARI, and the report handed to the patient, who returns to the referring ART center.

Drugs Used in the ARTC as per NACO guidelines for HIV-2 management are: Tenofovir 300mg + Lamivudine $300 \mathrm{mg}+$ Lopinavir $200 \mathrm{mg}+$ Ritonavir $50 \mathrm{mg}$. The cases are under regular follow-up of ART plus centres. HIV status of spouses and offspring is also investigated.

Improvement or deterioration in general well-being, weight, CD4 counts, haemoglobin, new opportunistic infections, side effects of drugs, adherences are considered for evaluation of therapy.

As viral load measurement is not yet available for HIV-2 infection in the Government setup, response to treatment has to be assessed clinically, by rise in CD 4 count and weight gain. The lack of large observational or randomized treatment studies in HIV-2-infected patients makes it difficult to assess when therapy should be started. Therapy needs to be started before advanced immunodeficiency develops. The recent U.S. Department of Health and Human Services (DHHS) guidelines recommend that in HIV-1-infected patients with a CD4 cell count $<500 / \mathrm{mm}$ therapy should be initiated, but they do not specifically comment on when ART should be initiated in HIV-2 infected patients. ${ }^{3}$ The 2008 French guidelines recommend ART initiation for all symptomatic patients, for HIV-infected pregnant women (to prevent transmission to the infant) and for asymptomatic patients who have a CD4 cell count $<350 / \mathrm{mm}^{3}$ This is the protocol followed by NACO, India too. Furthermore, these guidelines also suggest that therapy in HIV-2 should be considered when the CD4 cell count is $<500 / \mathrm{mm}^{3}$

Murugan and Anburajan observed a prevalence of $0.29 \%$ of HIV-2 infection in south Tamil Nadu. Soloman et al observed a prevalence of $0.8 \%$ of HIV-2 among urban population and $0.3 \%$ among rural population in Tamil Nadu.

The modes of transmission for HIV-2 are the same as those for HIV-1. HIV-2 is intrinsically resistant to nonnucleoside reverse transcriptase (NNRTI) drugs, Nevirapine and Efavirinz.

Drugs used in Government ART centres in India under the umbrella of NACO are Tenofovir 300mg + Lamivudine $300 \mathrm{mg}+$ Lopinavir $200 \mathrm{mg}+$ Ritonavir 50 $\mathrm{mg}$. It is observed that there is a significant rise in CD4 count and weight gain after initiation of second line ART as per NACO guidelines in cases of HIV 2 positive patients after at least one year of therapy. The general well-being also improves. Opportunistic infections that may be present are resolved after the initiation of therapy in patients who had received second line ART for more than a few months.

\section{Funding: No funding sources \\ Conflict of interest: None declared \\ Ethical approval: Not required}

\section{REFERENCES}

1. Harrison's Principles of Internal Medicine, $19^{\text {th }}$ edition. 2015;2:1216.

2. Campbell OT, Gandhi RT. Update on HIV-2 Infection. Oxford Journals, Medicine and Health, Clinical Infectious Diseases. 2011;53(6):780-7.

3. de Silva T, Weiss RA. HIV2 goes Global: an unaddressed issue in Indian antiretroviral programmes. Indian J Med Res. 2010;132(6):660-2.

4. http://www.aidsmap.com/HIV-2/page/1322993/.

5. Operational Guidelines for HIV Confirmatory Diagnosis, NACO, January, 2013. 
6. Agrawal S, Sawant S, Shastri J. Prevalence of HIV2 in Mumbai. Indian J Dermatol Venereol Leprol. 2010;76(6):709:10.

7. Kannangai R, Ramalingam S, Prakash KJ, Abraham OC, George R, Castillo RC. Molecular confirmation of human immunodeficiency virus (HIV) type 2 in HIV-seropositive subjects in south India. Clin Diagn Lab Immunol. 2000;7(6):987-9.
8. Ekouevi DK, Tchounga BK, Coffie PA, Tegbe J, Anderson AM, Gottlieb GS, et al. Antiretroviral therapy response among HIV-2 infected patients: a systematic review. BMC Infectious Dis. 2014;14:461. doi: 10.1186/1471-2334-14-461.

Cite this article as: Borkar MS, Kashid AA. HIV-2: an overview. Int J Res Dermatol 2015;1:7-9. 